UDC 572.524.3: $611.018+576.54+57.053$

Kalmukova O. O.

State Institute of Genetic and Regenerative Medicine NAMS, Kyiv, Ukraine

Educational and Scientific Centre "Institute of Biology", Taras Shevchenko National University of Kyiv, Kyiv, Ukraine

e-mail: olesyakalmukova@gmail.com

\title{
STEM CELLS IN NAIL UNIT OF MAMMALIANS
}

\section{ABSTRACT}

The review analyzes the current state of experimental studies on the ability to obtain and cultivate stem cells from the nail organ and their possible involvement in the regeneration of a limb. It has been known that the nail unit consists of a pool of undifferentiated cells which provide sustained growth and nail repair throughout life. But, nowadays the issue of stem cell niche localization in the nail organ remains unresolved. Also, researchers demonstrated involvement of these cells in the restoration of amputated limbs, in particular, through activation of certain signaling pathways (Wnt, BMP, Notch), and epithelial-mesenchymal interactions, but the detailed mechanism of this process is poorly understood. It is supposed that the nail organ has two sources of undifferentiated cells of different origin: the proximal nail fold and the dorsal

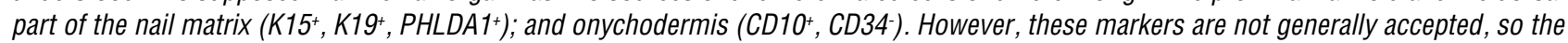
search for markers combinations for exhaustive and complete characterization of stem cells from the nail organ continues.

KEYWORDS: nail unit, stem cells, onychodermis, limb regeneration

The nails are structures that grow on the dorsal side of the fingers and contain a lot of hard keratins. Nails are derived formations of epithelial tissues, along with hair, hooves and claws. A number of foreign researchers call the nails as «nail organ» or a «nail unit» in an effort to emphasize the complexity of their structure and mechanisms of their functioning $[1$, 2]. The underestimation of the nail unit value among other organs was caused by insufficient knowledge about the biology of the nail. At present the latest research on the physiology of the nail revealed important biological aspects, including the presence of stem cells, complex signaling pathways of the regulation of nail differentiation and homeostasis, involvement of these pathways in damaged limb restoring, epithelialmesenchymal interactions between the nail epithelium and specific nail mesenchyme - onychodermis. In this regard, it is clear that the nail is a complex organ and has a more important role in the existence of the organism than previously thought, and therefore the use of the term «nail organ / unit» instead of the «nail» is more appropriate.

Currently there is a continuing search for new sources of stem cells for cell therapy, and it is the actual to consider the nail unit as a potential localization of undifferentiated cells as nails grow and renew throughout life.

Regeneration of limbs is often observed in invertebrates and lower vertebrates. In this case restoration of the structure is due to the differentiation of the pool of undifferentiated cells that form a regenerative blastema slightly below the amputation. In higher vertebrates, including mammals, limb regeneration occur in a different way. The volume of qualitative limb replacement depends on the extent of its damage, including with respect to the nail organ. Also there has been found a prominent role of stem cells from the nail organ in stimulating proliferation and synthesis of certain compounds (extracellular matrix, growth factors and others) by mesenchymal and bone cells.

Biology of stem cells from the nail unit is one of the fundamental questions. Compared with similar structures, such as hair follicle where stem cell niche location is already known - a bulge zone [3], the nail organ is poorly understood. Perhaps this is due to inaccessibility and invasiveness in obtaining materials for the research. The origin of these stem cells remains unidentified: from neural crest, epithelium or mesenchyme [4]. Knowledge obtained in the future will help to understand the pathogenesis and the possible ways of treating such diseases as vitiligo [5], psoriasis [6], onychomycosis [7] and others, which pathogenesis involves not only the immune system, but the disturbance in the functioning of melanocytes, keratinocytes and epithelial cells.

\section{ANATOMY AND HISTOLOGY OF THE NAIL ORGAN}

Nail unit and hair follicle are skin appendages, their structures have more in common than different [8]. Even in 1968 Achten G. proposed a hypothesis, which posits an analogy of these two structures: the nail organ can be compared to the hair follicle longitudinal section rotated by $90^{\circ}$ [9]. While anatomical analogy between hair follicles and nail matrix or between the nail and hair shaft is not in doubt, the equivalent of bulge with stem cells is still difficult to identify in the nail organ. 
The main components of the nail unit, proximal nail fold (PNF), two lateral and distal nail folds, nail matrix, nail bed and hyponychium, form and maintain the nail plate, keratinized corneous structure that continually grows throughout life. [10] Each component has a specific histological characteristic (Fig. 1):

- Proximal nail fold - wedge shaped skin invagination on the dorsal side of a finger; it has two surfaces that are histologically different: the dorsal surface is an extension of the finger skin with sweat glands, but without pilosebaceous units organization; and ventral surface has a thin epithelium without appendages and is closely adjacent to the dorsal surface of the nail plate.

- Cuticle is a continuation of the ventral surface of the PNF, consists of soft keratin and forms a corneous layer of a finger.

- $\quad$ Nail matrix begins right after the PNF. It is formed by thin stratified squamous epithelium without granular layer. Dorsally to the nail matrix there is an eosinophilic region - keratogenic zone where proliferating keratinocytes of the matrix begin to express hard keratin.

- Nail plate is formed as a result of the progressive spread of nail matrix cells as they are maturing. During this process, there is a disintegration of their nuclei and high levels of keratin accumulation.

- Nail bed extends from the distal end of the nail matrix (lunula) to hyponychium. It consists of a thin epithelium without a granular layer, which epithelial cells have a unique longitudinal location.

- Hyponychium, which covers the distal nail fold, is formed by normal epithelium with granular layer.

\section{EMBBRYOGENESIS OF THE NAIL ORGAN}

At the $10^{\text {th }}$ week of development there appears a primary nail field that is limited by proximal, distal and lateral grooves. In the proximal part of the primary nail field there is a primary primordial matrix that grows ventrally and, eventually, invaginates, forming PNF at the end of $13^{\text {th }}$ week. During the $14^{\text {th }}$ week there is a rapid differentiation of primordial matrix of the nail in the area of PNF, where formation of the nail plate begins. Along with the growth of the nail matrix the nail plate is growing too. By the end of the $17^{\text {th }}$ week it already covers all the nail bed. [14]

\section{THE DIFFERENCE BETWEEN NAIL ORGAN}

\section{STRUCTURE OF MICE AND HUMANS}

The availability of human material for research is limited, because the procedure of the nail unit biopsy is highly invasive and a person has only 20 nails. So the question arises about the similarities of murine and human nails. Sources of human nails may be abortive material or postoperative (e.g. after amputation extra fingers at the polydactyly).

Murine nail organ is commonly called a claw, and humans' one is a fingernail. In 1936 C. LeGros insisted on such differences in the name, seeking to emphasize their differences. [11] In support to his proposal, he cited several abstracts (Table 1).

It is clear that these differences show only anatomical difference, and functional components of the nail organ are the same in two species (Figure 2). Therefore, the distinction between «claws» and «nails» are rather arbitrary.

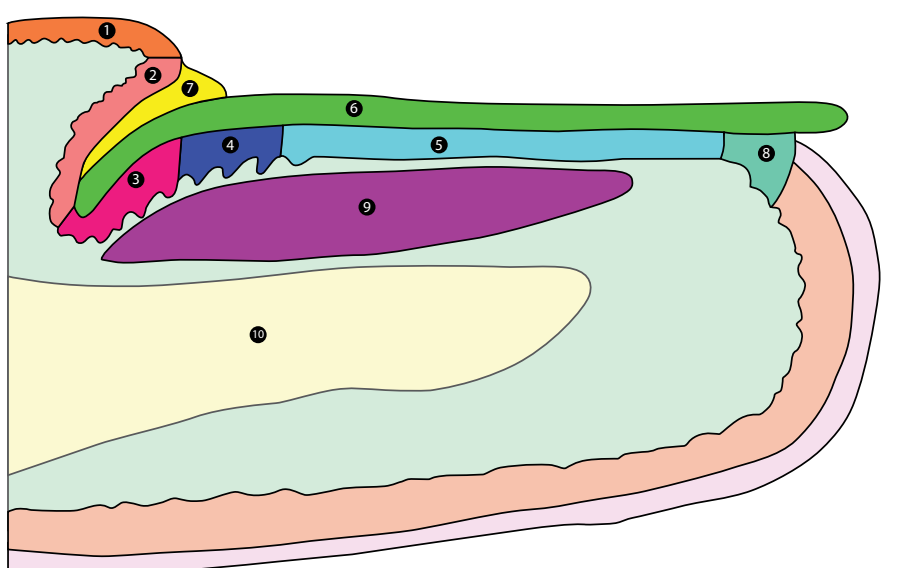

Fig. 1. The structure of the human nail organ: 1 - dorsal part of the proximal nail fold (PNF);

2 - ventral part of the PNF; 3 - dorsal part of the nail matrix;

4 - ventral part of the nail matrix; 5 - nail bed;

6 - nail plate; 7 - cuticle; 8 - hyponychium;

9 - onychodermis; 10 - distal phalanx.

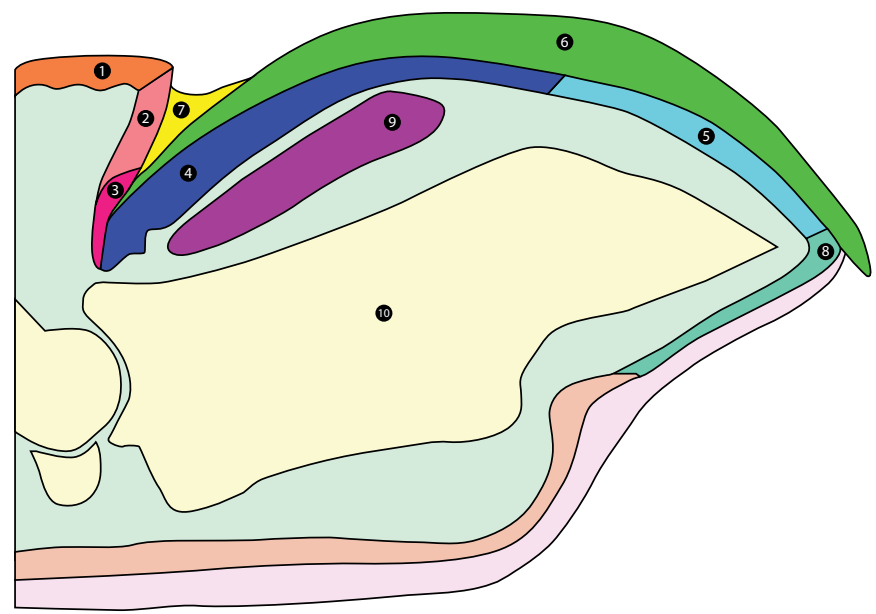

Fig. 2. The structure of the murine nail organ: 1 - dorsal part of the proximal nail fold (PNF):

2 - ventral part of the PNF; 3 - dorsal part of the nail matrix;

4 - ventral part of the nail matrix; 5 - nail bed;

6 - nail plate; 7 - cuticle; 8 - hyponychium;

9 - onychodermis; 10 - distal phalanx.
Table 1. Comparison of the human and murine nail structure. $\nabla$

\begin{tabular}{|c|c|c|}
\hline FEATURE & HUMAN & MOUSE \\
\hline The shape of the nail plate & Rounded & Sharpened \\
\hline Placement of the distal end of the nail plate & $\begin{array}{l}\text { Immersed in the ventral } \\
\text { groove }\end{array}$ & $\begin{array}{l}\text { Ends with flat } \\
\text { hyponychium }\end{array}$ \\
\hline The form of terminal phalanx bone & Convex & Sharpened \\
\hline The method of terminal phalanx connection & Movable & Still \\
\hline
\end{tabular}


To determine the similarity between the nail organ of mice and humans several researchers analyzed the level of soft and hard keratins expression using immunohistochemical study $[12,13]$. In the murine nail unit there were revealed local places of keratin expression (e.g. in the basal layer of the PNF ventral part - keratin 5, 14; in the suprabasal layer of the PNF ventral part $-6,10,14$; in the basal layer of the dorsal part of the matrix $-5,6,1417$, etc.) and the majority, but not all of them, are similar to those found in human nail organ (e.g. only a human has keratin 1 expression in the basal and subprabasal layers of the PNF ventral part and dorsal part of the matrix) [11]. The difference is observed in the nail bed due to different degrees of interaction of epithelium with dermal elements. In humans there is a deeper interaction as dermal papillae go deep into the epithelium; in mice, this interaction is less pronounced - derma looks like a dome that underlies the epithelium in the area of the nail bed.

Macroscopic, microscopic and immunohistochemical studies have shown that mice nail organ meets the basic characteristics of human nails. Therefore, all of the above makes murine nail apparatus suitable for the use as an experimental model object homologous to human nail organ.

\section{DIFFERENTIATION AND HOMEOSTASIS OF THE NAIL UNIT}

The development of the nail organ is closely linked by complex interactions of signal pathways of ectoderm and mesoderm, as differentiation of the nail organ (like a hair follicle) requires connection between the epidermis and mesenchymal structures under it. However, if the development is the same, the mechanisms of homeostasis are different because the hair follicle growth is cyclical in nature, including anagen (growth), catagen (involution), telogen (rest). Fingernails grow continuously and have a control mechanism of regulation between periods of growth and rest. In coordination of interactions between the epidermis and mesenchyme during induction and differentiation of the nail unit many signaling molecules and pathways are involved, three of them play a determining role: Wnt, BMP, Notch

Wnt-pathway regulates both embryogenesis and tissue homeostasis in adulthood. The canonical pathway consists of Wnt-ligands (R-spondin - RSPO), a complex of receptors Frizzled (FZD) and transcription co-activator $\beta$-catenin [15]. Mutations in the genes RSPO lead to anonychia and genetic defects of phalanges and nails development $[16,17,18]$; genes FZD - nail dystrophy [19, 20]; genes $\beta$-catenin - disorders in the nail formation [21]. Immunohistochemical studies have shown the presence of FZD receptors on the cell membranes of the basal layer of the nail matrix, while the ligand RSPO is secreted by dermal fibroblasts [22]. Also, Wnt/ß-catenin pathway plays a significant role at the early stages of limb regeneration, but at later stages of activation it is not critical [23].

BMP-pathway controls the amount of hard keratin synthesis by cells of the nail bed through the transcription factors Msx2 and Foxn1. Mutations in these genes lead to hyperplasia of the nail bed, formation of fragile (Foxn1) and brittle (Msx2) nails [24]. Accordingly, BMP-pathway has additional functions in the regulation of cyclic work of the basal layer cells to maintain homeostasis of the distal nail matrix and nail bed.

Notch-pathway refers to the contact-dependent signaling with limited proteolysis, which provides spatial lateral inhibition. Activation of this cascade by matrix metalloproteinases (MMP), which are secreted by mesenchymal cells, in the keratogenic nail area leads to the lengthening of the nail plate [25]. In addition, MMP can regulate the permeability of growth factors in the intercellular matrix and signaling molecules to their effectors. Thus, there is a mesenchymal control of ectodermal appendages differentiation.

\section{ONYCHODERIIIS}

Nail organ has specialized mesenchyme - onychodermis consisting of onychofibroblasts that are positive for CD10 (membrane bound matrix metal proteinase), do not express CD34 (a transmembrane protein, ligand for selecting, homing receptor) and Nestin; and have a low affinity for eosin. Onychodermis is directly under the nail matrix and nail bed and immunohistochemically it is different from the surrounding dermis of the nail unit. When staining with alcian blue the cells are positive for acidic polysaccharides (mucopolysaccharides, glycosaminoglycans) [26]. Onychodermis can be already distinguished at the end of the $2^{\text {nd }}$ trimester of embryonic development. At the beginning of development the entire finger dermis has a similar phenotype $\left(\mathrm{CD}_{10}{ }^{+}, \mathrm{CD} 34^{-}\right)$; but at the end of the $13^{\text {th }}$ week these markers are no longer expressed by mesenchyme, except onychodermis location, that keeps embryonic differentiation features throughout life, unlike ordinary connective finger tissue. A similar structure was not found in embryonic or adult hair follicle [27]. There is also an interesting evidence of finding onychodermis in the place of ectopic nails development [28], which further emphasizes the importance of epithelialmesenchymal interactions in the biology of the nail unit. According to the previous studies using model organotypic culture, onychofibroblasts, which were around the nail matrix cells, induced expression of hard keratins by keratinocytes not from the nail matrix through epithelial-mesenchymal interactions and the inclusion of BMP-signaling cascade [29].

\section{STEM CELLS FROM THE NAIL ORGAN}

In contrast to the hair follicle, where stem cell area location is well described $[30,31]$, the search for a stem cell niche in the nail organ continues. Location of these cells is suggested to be in the ventral surface of the PNF, nail bed and nail matrix.

Stem cells have certain features: these cells have a very slow cell cycle and can store an exogenously introduced label in the core for several weeks. This is a basis for a study on the inclusion of bromine deoxyuridine (BrdU) in the nucleus of the nail organ cells followed by immunohistochemical detection of cells that retain it for a long time. It was found that these cells are in the basal layer part of the nail matrix, which is adjacent to the nail bed (distal part of the matrix), i.e. in the middle area of the nail unit [22].

Another approach compared the size of the colony-forming units (CFU) of the basal layer cells of the nail matrix and the nail bed. The largest CFU size is showed by cells of the proximal part of the nail matrix, which is adjacent to the end of the ventral surface of the PNF [21].

Since the hair follicle and nail organ are epithelial derivatives, there were attempts to paint immunohistochemically for proteins that are markers of cells from the bulge zone (cytokeratins 15, 19, PHLDA1 - pleckstrin-homology-like domain, family $A$, and member 1 molecule involved in regulation of apoptosis). As a result, ventral surface area of PNF had high expression in all markers. Therefore the ventral surface is equivalent to PNF bulge zone, and may well be a place for the location of stem cells from of the nail unit [32]. In addition, staining for Ki-67 (marker of cells that actively proliferate) showed its high expression in cells of the nail matrix and a low one in the area of the ventral surface of the PNF. For comparison, cells from the bulge are negative for $\mathrm{Ki}-67$, because stem cells rarely divide [33].

Accordingly, a later method was used to detect cells that retain a label to identify stem cells in the basal layer of the PNF. There has been demonstrated location of these cells in a 3D ring volume structure, organized around the base of the nail. This cell population expresses markers of the hair follicle stem cells, is positive for keratin 15 and contributes to the structure maintenance of both the nail organ and epidermis, which is located nearby. Under normal conditions these cells are bifunctional, give rise to keratinocytes and epithelial cells. But during the trauma they are able to redirect the flow of stem cells in response to injury, through signaling pathways Wnt, BMP and to onychodermis. There has been proven differentiation of these cells through an intermediate state (cells with a moderate rate of cell cycle) into cells of the nail matrix (high speed cell division) [34].

\section{CULTIVATION OF CELLS FROM THE NAIL ORGAN}

It is relevant today to search for methods of selection in culture and cells markers from the nail organ, including undifferentiated stem cells. The first attempts were made in 1992, by two groups of scientists - Picar- 
do M., et al. and Kitahara T., et al. - who were study expressing hard (hair-type keratins) and soft (skin-type keratins) keratins by nail organ cells. They obtained a cell culture from human nail matrix, namely from its ventral part $[35,36]$. As a result there was analyzed the morphology of these cells on the ultrastructural level. It was found that compared with epidermal keratinocytes, the cells from nail matrix are larger in size, have a high ratio of euchromatin to heterochromatin, low nuclear-cytoplasmic ratio and high growth rate [37]. There was also shown solid expression of keratin (AE13 antibody against highly sulfated proteins) exactly by cells from the ventral part of the nail matrix, and consequently its derivatives, so it was possible to clearly separate the nail matrix cells from normal epithelial keratinocytes. In this regard, hard keratins in relation to the cells from nail matrix are called "keratins related to differentiation». It is proved that in the culture these cells begin expressing hard keratins at a concentration of $0.15 \mathrm{mM}$ calcium, they are at the stage of differentiation; whereas at calcium concentration below $0.1 \mathrm{mM}$ expression was not observed. Therefore, the differentiation of cells from the nail matrix can be controlled by changing the concentration of calcium outside.

Later cells from nail matrix of bull hooves and mice claws were introduced in cultures $[38,39]$. By the expression of keratins in the nail matrix 3 cell types are distinguished: ones that synthesize soft keratins 10/11 (dorsal part of the nail matrix), hard ones (ventral part of the nail matrix) and cells that synthesize 2 types of keratins simultaneously (apical part of the nail matrix) [40]. The cells of the apical parts are differentiation intermediaries between cells from dorsal and ventral parts. At culturing cells from the ventral part of the nail matrix, which synthesize hard keratins in vivo, some cells express soft keratins. This indicates that some of the cells change their direction of differentiation, replacing those parts of nail matrix, which are missing in vitro.

Recent studies are aimed at the search for markers of stem cells from the nail organ and the development of protocols for these cells allocation [21, 41].

\section{NAIL ORGAN AND PROSPECTS FOR REGENERATIVE MEDICINE}

According to the recent studies, not only amphibians and reptiles are able to restore damaged limbs, but mammals under certain conditions. This regeneration process requires the presence of the nail organ. The degree and completeness of the recovery depends on the level of amputation against the nail organ: at amputation of the distal phalanx of the limb there is observed almost complete reproduction of integrity and original form of normal intact limb. The limit at which keeps perfect regeneration is the midline between the proximal end of the distal phalanx and the middle part of the finger nail matrix [42]. Also, there was shown correlation between the preservation of the nail unit and the regeneration of amputated bone. In the experiments of Zhao $\mathrm{W}$ et al. the ends of mice phalanxes were amputated at different levels (distal - a small portion of the terminal phalanx and proximal all terminal phalanx) so that the nail was removed completely or partially preserved. In the absence of the nail the bone did not grow even at the distal level damage. Conversely, when nails were retained, bones restored after damage at the proximal level [43].
The regeneration of limbs in lower vertebrates is due to the formation of blastema - a pool of homologous poorly differentiated cells which are formed by differentiation (cell partially remains committed, but restores the ability to mitosis) near the place of injury, proliferate in a specific area of reproduction (differentiated cells grow on top, and blastema remains at a permanent place), followed by redifferentiation (redifferentiation of the cells of the same kind, such as in tailed amphibians), transdifferentiation (in other cells of the same germ layers, for example, in newt, axolotl) and metaplasia (cells into different germ layers, for example in nemertea, ascidians).

In higher vertebrates, regeneration of limb also occurs due to the pool of poorly differentiated cells that express embryonic genes, but this cell population is heterogeneous (onychofibroblasts, endothelial progenitors, osteoblasts, stem cells from the PNF, nail matrix). These cells are tissue residents, they are clearly committed according to their belonging to germ layers, and restore only the damaged parts, which are their derivatives. [44] The epithelium of the nail organ organizes their work using signaling molecules [1], because an amputated proximal phalanx grows back after transplantation of the nail organ part [45]. The success of limb regeneration depends on the level of the finger amputation and requires the presence of onychodermis [46]. Most of blastema cells express stem cells marker Sca-1 and endothelial marker CD31 [47]. Also, it was shown that Wnt-signaling pathway is disrupted, and bone regeneration stops at inclusion of $\beta$-katenin in the nail organ cells [21]. After amputation Wntpathway is activated in nail progenitor cells, while mesenchymal cells proliferate and express Runx2, a key transcription factor that is associated with the differentiation of osteoblasts.

In addition to Wnt-pathway BMP-signaling cascade plays a role in regeneration. After amputation, there is a decrease in expression of two inhibitors of BMP cascade Bambi i Decorin in all cells of the nail organ that increases the synthesis of BMP (such as BMP-4), and as a result, Msx-1 and Msx-2, thereby enabling them to improve proliferation of both nail organ cells and onychodermis [34, 48, 49]. Msx-1-positive onychodermis during regeneration acts as a signaling center that produces the required paracrine factors [46]. It is proved that targeted therapy, which activates BMP-2 and BMP-7, stimulates limb recovery after phalanx damage at the proximal level [50].

In tailed amphibians (salamanders) successful regeneration depends on the mitogenic signals which are produced by nerve cells. In contrast, the recovery of the finger end in mice happens even in the absence of nerve fibers, indicating the other sources of signaling molecules (PNF, nail matrix, onychodermis), but sometimes there can be observed defects in phalanx bones (disorganized structure of the bone instead of trabecular) and nail matrix (hypertrophy), for example, in patients with spinal cord injuries [51].

For induction of phalanx bone formation there are required both Wntactivated cells of the nail matrix and Msx-1-positive onychodermis [52]. Such epithelial-mesenchymal interaction affects the activation of Wnt, Notch and BMP-signaling pathways in future osteoblasts through activation of gene Runx2 [53].

\section{CONCLUSION}

Nail organ has two sources of undifferentiated cells of different origin: proximal nail fold, dorsal part of the nail matrix and onychodermis. A unified set of markers for identification the stem cells from the nail organ remains undetermined. Studies on mechanisms of their interaction between themselves and the impact they have on surrounding tissue during the restoration of the damaged limb continue. Further study of stem cells from the nail unit is promising for the development of regenerative medicine. 
1. Saito M, Ohyama M, Amagai M. Exploring the biology of the nail: An intriguing but less-investigated skin appendage. J Dermatol Sci. 2015. Available: http://dx.doi. org/10.1016/j.jdermsci.2015.04.011

2. De Berker D, Angus B. Proliferative compartments in the normal nail unit. 1996; 135: 555-59.

3. $\quad$ Myung $P$, Ito $M$. Dissecting the bulge in hair regeneration. J Clin Invest. 2012; 122: 448-54.

4. Naveau A, Seidel K, Klein OD. Tooth, hair and claw: comparing epithelial stem cell niches of ectodermal appendages. Exp Cell Res. 2014; 325(2): 96-103.

5. lannella G, Greco A, Didona D, et al. Vitiligo: Pathogenesis, clinical variants and treatment approaches Autoimmun Rev. 2015. Available: http://dx.doi.org/10.1016/j. autrev.2015.12.006

6. Wcisło-Dziadecka D, Zbiciak-Nylec M, Brzezińska-Wcisło L, et al. TNF-a in a molecularly targeted therap of psoriasis and psoriatic arthritis. Postgrad Med J. 2015. Available: doi:10.1136/postgradmedj-2015-133419

7. Tucker JrJ. Nail Deformities and Injuries Prim Care Clin Office Pract. 2015. Available: http://dx.doi.org/10.1016/j.pop.2015.08.005

8. Baran R, Dawber RP, Haneke E. Hair and nail relationship. Skinmed. 2005; 4: 18-23.

9. Achten G. Normale Histologie und Histochemie des Nagels. In: Handbuch der Haut- und Geschlechtskrankheiten, Bandl/1 (Jadassohn, J., Hrsg.), Berlin-HeidelbergNew-York: Springer, 1968. P. 339-76.

10. De Berker DAR, Baran R. Baran \& Dawber's diseases of the nails and their management. 4th ed, Oxford, England: Wiley-Blackwell, 2012. $832 \mathrm{p}$.

11. LeGros CWE. The problem of the claw in primates. Proc Zool Soc. 1936; 1:1-24.

12. Fleckman P, Karin J, Silva KA, et al. Comparative Anatomy of Mouse and Human Nail Units. Anat Rec (Hoboken). 2013; 296(3): 521-32.

13. Perrin C, Langbein L, Schweizer J. Expression of hair keratins in the adult nail unit: an immunohistochemical analysis of the onychogenesis in the proximal nail fold, matrix and nail bed. British Journal of Dermatology. 2004; 151: 362-71.

14. Zaias N. Embryology of the human nail. Arch Dermatol. 1963; 87: 37-53.

15. Saito M, Tucker DK, Kohlhorst D, et al. Classical and desmosomal cadherins at a glance. J Cell Sci. 2012; 125: 2547-52.

16. Blaydon DC, Ishii $Y, O^{\prime}$ Toole EA, et al. The gene encoding R-spondin 4 (RSPO4), a secreted protein implicated in Wnt signaling, is mutated in inherited anonychia. Nat Genet. 2006; 38: 1245-47.

17. Bergmann C, Senderek J, Anhuf D, et al. Mutations in the gene encoding the Wnt-signaling component R-spondin 4 (RSPO4) cause autosomal recessive anonychia. Am J Hum Genet. 2006; 79: 1105-9.

18. Aoki $M$, Kiyonari $H$, Nakamura $H$, et al. R-spondin2 expression in the apical ectodermal ridge is essential for outgrowth and patterning in mouse limb development . Dev Growth Differ. 2008; 50: 85-95.

19. Wilson NJ, Hansen $C D$, Azkur $D$, et al. Recessive mutations in the gene encoding frizzled 6 cause twenty nail dystrophy - expanding the differential diagnosis for pachyonychia congenita. J Dermatol Sci. 2013; 70: 58-60.

20. Naz G, Pasternack SM, Perrin C, et al. FZD6 encoding the Wnt receptor frizzled 6 is mutated in autosomal-recessive nail dysplasia. Br J Dermatol. 2012; 166: 1088-94.

21. Takeo M, Chou WC, Sun Q, et al. Wnt activation in nail epithelium couples nail growth to digit regeneration. Nature. 2013; 499: $228-32$.

22. Nakamura $M$, Ishikawa $O$. The localization of label-retaining cells in mouse nails. J Invest Dermatol. 2008; 128: 728-30.

23. Yokoyama H, Ogino H, Stoick-Cooper CL, et al. Wnt/ $\beta$-catenin signaling has an essential role in the initiation of limb regeneration. Dev Biol. 2007; 306(1): 170-78.

24. Cai J, Ma L. Msx2 and Foxn1 regulate nail homeostasis Genesis. 2011; 49: 449-59.

25. Lin MH, Kopan R. Long-range, nonautonomous effects of activated Notch1 on tissue homeostasis in the nail. Dev Biol. 2003; 263: 343-59.

26. Lee DY, Park JH, Shin HT, et al. The presence and localization of onychodermis (specialized nail mesenchyme) containing onychofibroblasts in the nail unit: a morphological and immunohistochemical study Histopathology. 2012. Available: DOl: 10.1111/j.1365-2559.2012.04210.x.

27. Sellheyer $K$, Nelson $P$. The concept of the onychodermis (specialized nail mesenchyme): an embryological assessment and a comparative analysis with the hair follicle. J Cutan Pathol. 2013; 40: 463-71.

28. Park JH, Kim JH, Lee JH, et al. Onychodermis (specialized nail mesenchyme) is present in ectopic nails J Cutan Pathol. 2013; 40: 600-02.

29. Okazaki M, Yoshimura K, Fujiwara H, et al. Induction of hard keratin expression in non-nail-matrical keratinocytes by nail-matrical fibroblasts through epithelialmesenchymal interactions . Plast Reconstr Surg. 2003; 111: 286-90.

30. Ohyama M, Terunuma A, Tock CL, et al. Characterization and isolation of stem cell-enriched human hair follicle bulge cells. J Clin Invest. 2006; 116: 249-60.

31. Ohyama M. Hair follicle bulge: a fascinating reservoir of epithelial stem cells. J Dermatol Sci. 2007; 46: 81-9.

32. Sellheyer K. Nail stem cells. JDDG. 2013; 1103: 235-39.

33. Sellheyer $K$, Nelson $P$. The ventral proximal nail fold: stem cell niche of the nail and equivalent to the follicular bulge—a study on developing human skin. 2012; 39 : $835-43$.

34. Leung $Y$, Kandyba $E$, Chen $Y B$, et al. Bifunctional ectodermal stem cells around the nail display dual fate homeostasis and adaptive wounding response toward nail regeneration. Proc Natl Acad Sci USA. 2014; 111: 15114-19.

35. Kitahara T, Ogawa H. Cultured nail keratinocytes express hard keratins characteristic of nail and hair in vivo. Arch.Dermatol. Res. 1992; 284: 253-56.

36. Picardo M, Marchese C, Zompetta C, et al. Characterization of human nail matrix cells in vitro [Abstract]. J Invest Dermatol. 1992; 98: 523A.

37. Picardo M, Tosti A, Marchese C, et al. Characterization of cultured nail matrix cells . Journal of the American Academy of Dermatology. 1994; 30(3): 434-40.

38. Kitahara T, Ogawa H. Coexpression of keratins characteristic of skin and hair differentiation in nail cells. J. Invest. Dermatol. 1993; 100: 171-75.

39. Kitahara T, Ogawa H. Variation of differentiation in nail and bovine hoof cells. J. Invest. Dermatol. 1994; 102: 725-29.

40. Kitahara T, Ogawa H. Cellular Features of Differentiation in the Nail. Microscopy research and technique. 1997; 38: 436-42.

41. Lehoczky JA, Tabin CJ. Lgr6 marks nail stem cells and is required for digit tip regeneration. PNAS. 2015; 112(43): 13249-254.

42. Borgens RB. Mice Regrow the Tips of Their Foretoes. Borgens Sciense. 1982; 217: 747-50.

43. Zhao W, Neufeld DA. Bone regrowth in young mice stimulated by nail organ. Exp Zoolog. 1995; 271: 155-59.

44. Rinkevich $Y$, Lindau $P$, Ueno $H$, et al. Germ and lineage restricted stem/progenitors regenerate the mouse digit tip. Nature. 2013; 476(7361): 409-13.

45. Mohammad KS, Day FA, Neufeld DA. Bone growth is induced by nail transplantation in amputated proximal phalanges. Calcif Tissue Int. 1999; 65: 408-10. 
46. Lehoczky JA, Robert B, Tabin CJ. Mouse digit tip regeneration is mediated by fate-restricted progenitor cells. PNAS. 2011; 108(51): 20609-614.

47. Fernando WA, Leininger E, Simkin J, et al. Wound healing and blastema formation in regenerating digit tips of adult mice. Dev Biol. 2011; 350(2): 301-10.

48. Han M, Yang X, Lee J, et al. Development and regeneration of the neonatal digit tip in mice. Dev Biol. 2008; 315: 125-35.

49. Tamura K, Ohgo S, Yokoyama HA. Limb blastema cell: A stem cell for morphological Regeneration. Develop. Growth Differ. 2010; 52: 89-99.

50. Yu L, Han M, Yan M, et al. BMP signaling induces digit regeneration in neonatal mice Development. 2010; 137: 551-9.

51. Rinkevicha $Y$, Montorob DT, Muhonenb E, et al. Clonal analysis reveals nerve-dependent and independent roles on mammalian hind limb tissue maintenance and regeneration. PNAS. 2014; 111(27): 9846-51.

52. Rinkevich Y, Maan ZN, Walmsley GG, et al. Injuries to appendage extremities and digit tips: a clinical and cellular update Developmental Dynamics. 2015. Available: DOI 10.1002/dvdy.24265

53. Lin GL, Hankenson KD. Integration of BMP, Wnt, and Notch signaling pathways in osteoblast differentiation. J Cell Biochem. 2011; 112(12): $3491-501$.

\begin{tabular}{|c|c|}
\hline TRTILE ON THE SITE \\
\hline TRANSPANTOLOG.ORG \\
\hline
\end{tabular}

The author indicates no potential conflicts of interest.

Received: March 14, 2016

Accepted: May 11, 2016 